\title{
Towards broadspectrum antiprotozoal agents
}

\author{
Kelly Chibale* \\ Department of Chemistry, University of Cape Town, Rondebosch 7701, South Africa \\ E-mail: chibale@science.uct.ac.za
}

\section{Dedicated with great respect and admiration to Professor James R. Bull on the occasion of his retirement}

\begin{abstract}
Since the market for antiparasitic drugs is in poor Third World countries, innovative and cost effective approaches to antiparasite drug discovery and development are urgently needed. Development of broadspectrum antiprotozoal agents acting against multiple protozoan parasites offers numerous advantages in addressing the challenges inherent in the aforementioned approaches. With a view to addressing these challenges, our research group at the University of Cape Town has initiated research projects whose main objectives include: (i) the development of single agents that provide target-directed inhibition of multiple protozoans; (ii) the development of single agents that provide maximal antiprotozoan activity by acting against multiple parasitic targets. The human diseases of leishmaniasis, malaria and trypanosomiasis have been selected for proof of concept studies with cysteine proteases and oxidoreductases present in the respective parasites selected as enzyme targets for antileishmanial, antimalarial and antitrypanosomal drug discovery efforts.
\end{abstract}

Keywords: Cysteine proteases, trypanothione reductase, trypanosomiasis, leishmaniasis, malaria

\section{Introduction}

Current chemotherapy for malaria, trypanosomiasis and leishmaniasis is inadequate, toxic or both. While potential demand for antiparasitic drugs in endemic areas is high, drug resistance to established drugs is a major problem. Previous first line drugs have been rendered completely ineffective in most endemic areas. Understanding of the mechanism of resistance and development of chemical agents without cross-resistance to existing drugs is of paramount importance. Since the market for antiparasitic drugs is in poor Third World countries, innovative and cost effective approaches to antiparasite drug discovery and development are urgently needed. 
New biological targets on which to base new treatment strategies and which need to be exploited, have been identified and validated in recent years. Our research group at the University of Cape Town (UCT) is involved in the exploitation of some of these targets with the ultimate goal of potentially slowing down the development of drug resistance by identifying inhibitory molecules that affect at least two different targets within the same parasite as well as the development of common inhibitors and/or chemical scaffolds which may be used against multiple parasites. The approach we have taken is based on the following criteria: (a) some enzyme targets are commonly found in different parasites, (b) some enzyme targets are related across different parasites, (c) the catalytic mechanisms in the respective target enzyme families are similar, (d) much is known about structure/function relationships as well as selectivity towards parasite targets over the homologues in human families for some of the targets. We have focused on parasitic cysteine proteases and oxidoreductases as enzyme targets.

Cysteine proteases (CPs) and the disulfide oxidoreductases [trypanothione reductase (TryR), glutathione reductase (GR) and thioredoxin reductase (TrxR)] exemplify common enzyme targets present in different parasites [criteria (a)]. CPs and TryR have been identified in Trypanosoma brucei Trypanosoma cruzi and Leishmania. ${ }^{1-6}$ Although malaria parasites do not contain TryR, they contain $\mathrm{CPs}^{1-3}$, GR (Plasmodium falciparum GR, PfGR) and TrxR (Plasmodium falciparum TrxR, PfTrxR). ${ }^{6}$ Leishmanial (cathepsins $\mathrm{B}$ and L), malarial (falcipains) and trypanosomal (cruzain and rhodesain) CPs are related across the different parasites [criteria (b)]. Thiols in the form of active site cysteines are important in the catalytic mechanisms of both CPs and the oxidoreductases [criteria (c)]. For example TryR has a redoxactive cysteine pair and is known to be alkylated at Cys-53, an active site nucleophile implicated in an attack on the trypanothione disulfide moiety during the enzymatic reduction, by the thiolspecific reagent iodoacetamide, ${ }^{7,8}$ Fig. (1). This mechanism is somewhat similar in nature to that of PfGR and PfTrxR. In PfTrxR, the two C-terminal cysteine residues have been shown to be involved in catalysis presumably by an intramolecular disulfide/dithiol interchange with the redox-active cysteines at the active site. ${ }^{9}$ CPs utilize an active site cysteine thiol for hydrolysis. ${ }^{2}$ Much is known about structure/function relationships for example in inhibitors of haemozoin formation and TryR respectively [criteria (d)]. ${ }^{10-13}$

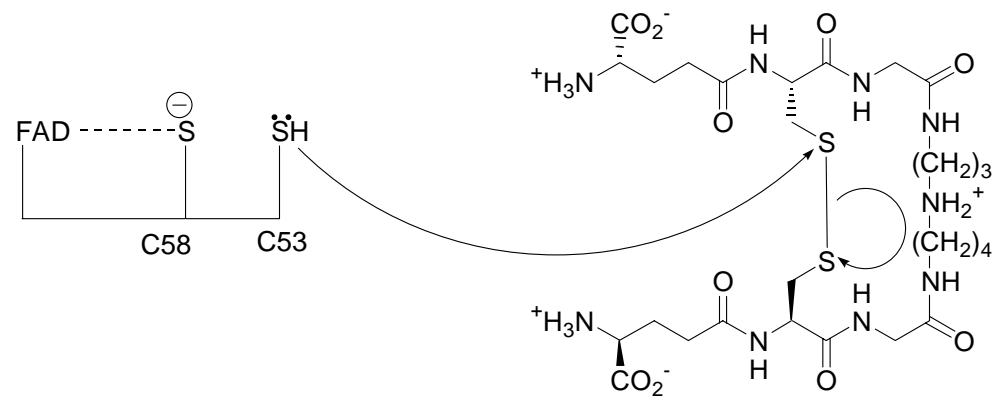

Trypanothione disulfide

Figure 1. Nucleophilic attack of Cys-53 on trypanothione disulfide during enzymatic reduction. 
CPs in protozoan parasites are attractive targets for the development of antiparasitic chemotherapy due to the key role they play at various stages in the life cycle of the parasites. On the other hand, the presence of enzymes of parasite thiol metabolism in trypanosomatids (TryR) and malaria parasites (PfGR and PfTrxR) is crucial to the maintenance of an intracellular reducing environment as a means of defence against oxidative stress. It has also recently been demonstrated that PfTrxR is an essential protein for erythrocytic stages of the malaria parasites. ${ }^{14}$ Thus these enzymes too present themselves as vulnerable targets for antiparasitic chemotherapy. On the other hand, haem, a product of the catabolism of haemoglobin and toxic to the malaria parasite, is also an attractive target for antimalarial drugs. ${ }^{15}$ Inhibition of PfGR can reduce the glutathione concentration necessary for parasite survival. A bi-therapeutic strategy in which haemozoin formation inhibiting quinoline moieties are combined with inhibitors of PfGR through ester prodrugs has recently been reported. ${ }^{16}$

Thus in view of the background above, it is reasonable to assume that common inhibitors affecting different targets within the same or different parasites could be developed. Covalent inhibitors which are able to alkylate thiol moieties in the aforementioned enzymes have potential to inhibit different enzymes from different parasites reversibly or irreversibly. Moreover, it is also reasonable to envisage common inhibitors that affect at least two different biological targets within the same parasite since, for example, trypanosomes and Leishmania contain both CPs and TryR. Aminoquinolines and aminoacridines which also target haem in malaria parasites have great potential as inhibitors of TryR since both chloroquine (Fairlamb, A. H., personal communication) and quinacrine ${ }^{13}$ are known inhibitors of TryR. While there maybe concerns about specificity, selectivity and how parallel improvement against two targets could be designed for in the case of common inhibitors affecting at least two different targets in the same parasite, the availability of 3D structures of some of the target proteins in complex with inhibitors on one hand, and structure-function relationships on the other, could go a long way in addressing such concerns.

\section{Reactions catalyzed by cysteine proteases}

The proposed general catalytic mechanism for CPs, in which an active site cysteine thiol is utilized for hydrolysis, is shown in Scheme 1. A histidine (His) residue polarizes the enzymecysteine thiol group of Cys (Enz-Cys) allowing deprotonation even under nearly neutral to weakly acidic $\mathrm{pH}$ conditions. The resulting highly nucleophilic thiolate/imidazolium ion pair allows the thiolate anion to attack the carbonyl carbon of the scissile amide bond resulting in a tetrahedral intermediate. This is followed by acylation of the enzyme and the liberation of the first product. A second tetrahedral intermediate is then formed via hydrolysis of the acylenzyme. The enzyme is regenerated following collapse of the second tetrahedral intermediate and product release. 

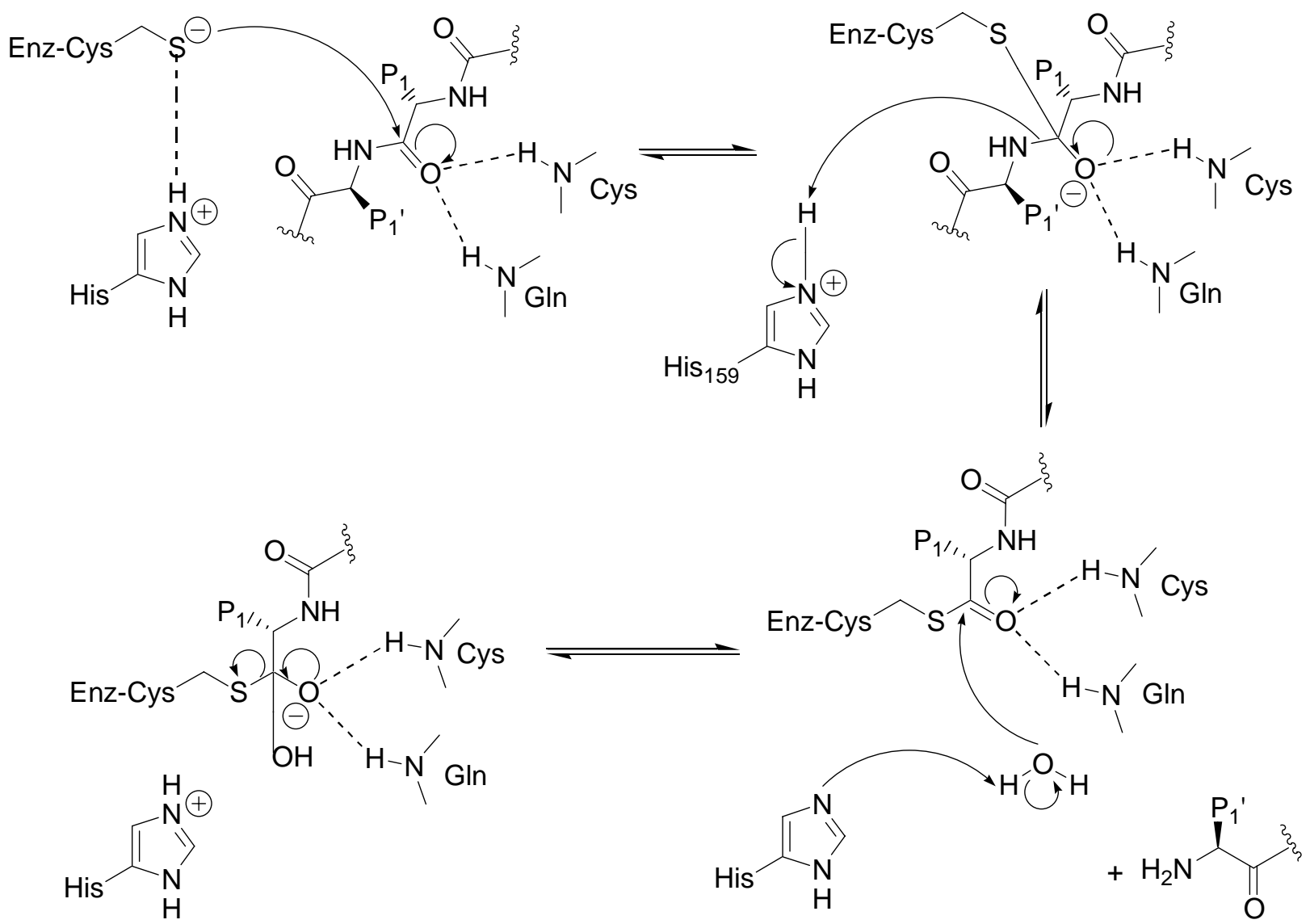

Scheme 1. Proposed catalytic mechanism for cysteine proteases.

\section{Reactions catalyzed by trypanothione and glutathione reductase}

On the other hand while most organisms utilize glutathione within their cells to maintain an intracellular reducing environment, Leishmania and the trypanosomes uniquely utilize trypanothione [ $N^{1}, N^{8}$-bis(glutathionyl)spermidine] as the antioxidant molecule. TryR maintains the required levels of trypanothione by catalyzing the NADPH-dependent reduction of its substrate trypanothione disulfide, Scheme 2. The human homolog, glutathione reductase (hGR) plays a similar catalytic role, Scheme 3. However, TryR and hGR are mutually exclusive for their respective substrates, trypanothione disulfide for TryR and glutathione disulfide for hGR. Despite playing identical roles, the two major factors accounting for the selectivity of inhibitors against the respective enzyme are charge and size. TryR has a negatively charged active site that requires a positively charged amino chain in the substrate or inhibitor. On the other hand, hGR has an overall positive charge to accommodate a negatively charged substrate or inhibitor. Concerning size, TryR has a larger active site compared to hGR in order to accommodate its larger substrate. 


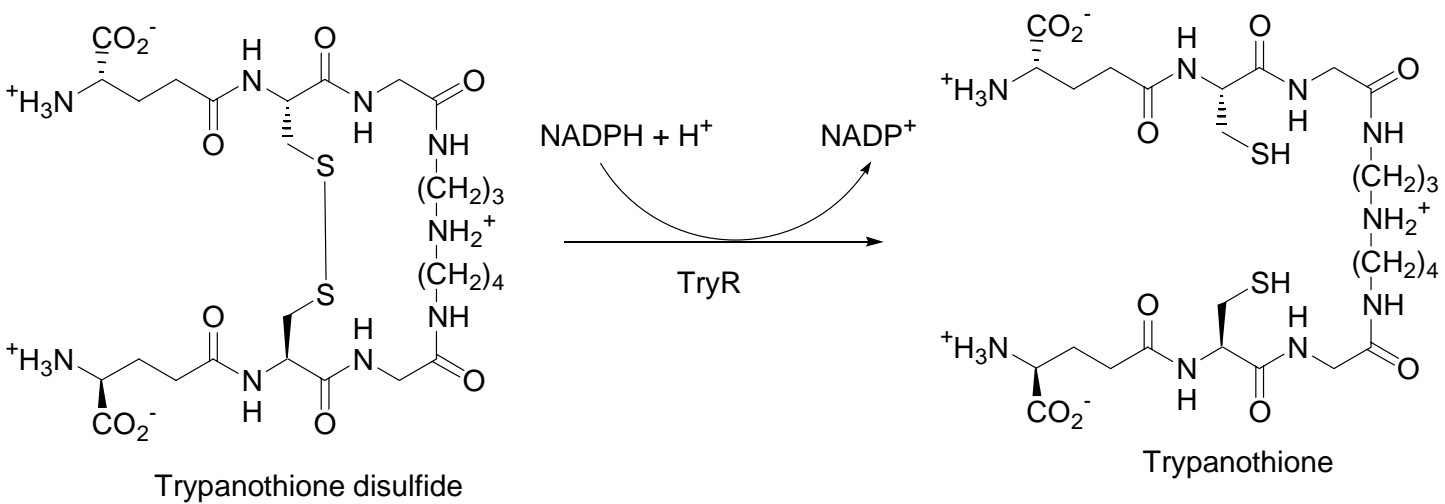

Scheme 2. Reaction catalyzed by trypanothione reductase.

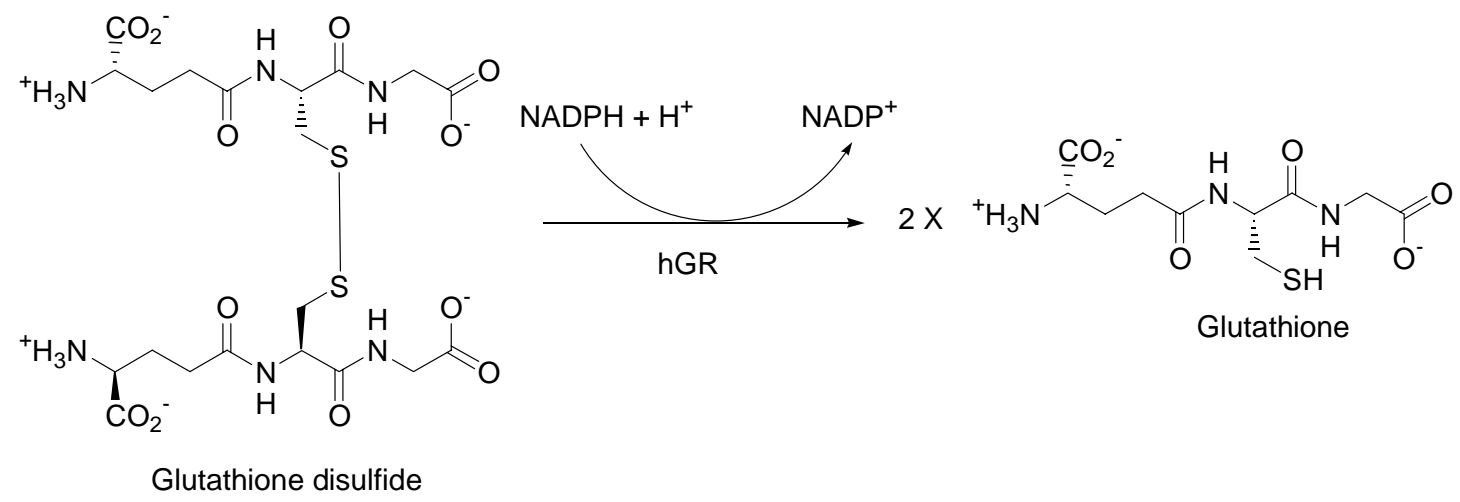

Scheme 3. Reaction catalyzed by glutathione reductase.

\section{Acknowledgements}

I would like to thank The Wellcome Trust for an International Research Development Fellowship award (grant number 052075/Z/97). Further financial support from the National Research Foundation of South Africa is gratefully acknowledged.

\section{References}

1. McKerrow, J. H. Int. J. Parasitol. 1999, 29, 833.

2. Sajid, M.; McKerrow, J. H. Mol. Biochem. Parasitol. 2002, 120, 1.

3. Rosenthal, P. J. Adv. Parasitol. 1999, 43, 105.

4. Fairlamb, A. H., Cerami, A. kinetoplastida. Annu. Rev. Microbiol. 1992, 46, 695.

5. Schirmer, R. H.; Muller, J. G., Krauth-Siegel, R. L. Angew. Chem., Int. Ed. 1995, 34, 141.

6. Krauth-Siegel, R. L.; Coombs, G. H. Parasitol. Today 1999, 15, 404. 
7. Borges, A.; Cunningham, M.; Tovar, J.; Fairlamb, A. H.. Eur. J. Biochem. 1995, 228, 745.

8. Krauth-Siegel, R. L.; Enders, B.; Henderson, G. B.; Fairlamb, A. H.; Schirmer, R. H. Eur J. Biochem. 1987, 164, 123.

9. Wang, P. F.; Arscott, L. D.; Gilberger, T. W.; Mueller, S.; Williams, C. H. Biochemistry 1999, 38, 3187.

10. De, D.; Krogstad, F. M.; Byers, L. D.; Krogstad, D. J. J. Med. Chem. 1998, 41, 4918.

11. Egan, T. J.; Hunter, R.; Kaschula, C. H.; Marques, H. M.; Misplon, A.; Walden, J. J. Med. Chem. 2000, 43, 283.

12. Faerman, C. H.; Savvides, S. N.; Strikland, C.; Breidenbach, M. A.; Ponasik, J. A.; Ganem, B.; Ripoli, D.; Krauth-Siegel, R. L.; Karplus, P. A. Bioorg. Med. Chem. 1996, 4, 1247.

13. Bonse, S.; Santelli-Rouvier, C.; Barbe, J.; Krauth-Siegel, R. L. J. Med. Chem. 1999, 42, 5448.

14. Krnajski, Z., Gilberger, T.W., Walter, R.D., Cowman, A.F.; Müller, S. J. Biol. Chem. 2002, 277, 25970.

15. Ziegler, J.; Linck, R.; Wright, D. W. Curr. Med. Chem. 2001, 8, 171.

16. Davioud-Charvet, E.; Delarue, S.; Biot, C.; Schwobel, B.; Boehmeme, C. C.; Mussigbrodt, A.; Maes, L.; Grellier, P.; Sergheraert, C.; Schirmer, H.; Becker, K. J. Med. Chem. 2001, 44, 4268. 